\title{
DIAGNOSTIC ACCURACY OF MODIFIED EARLY OBSTETRIC WARNING SYSTEM FOR PREDICTION OF MATERNAL MORBIDITY
}

\author{
Sadaf Zohra, Nayla Khalid, Uzma Urooj, Sumaira Khan, Asifa Siraj, Munawar Jannat Rana \\ Pak Emirates Military Hospital/National University of Medical Sciences (NUMS) Rawalpindi Pakistan
}

\begin{abstract}
Objective: To determine the diagnostic accuracy of modified early obstetric warning system for predicting maternal morbidity keeping ICU admission as "gold standard".

Study Design: Cross sectional validation study.

Place and Duration of Study: Department of Obstetrics and Gynecology, Pak Emirates Military Hospital, Rawalpindi, from Oct 2018 to Apr 2019.

Methodology: A total of 205 patients of singleton pregnancy, post-natal period $<6$ weeks, history of hypertension and diabetes were considered in the study. Women were followed up to 6 weeks for maternal morbidity in term of intensive care admission. All the data regarding findings of Modified early obstetric warning system and intensive care admission was noted.

Results: The age range of patients was 18-40 years with mean age of $31.585 \pm 3.31$ years, mean gestational age was 37.595 \pm 1.84 weeks and mean parity was $1.839 \pm 1.59$. Modified early obstetrics warning system as shown sensitivity of $84.38 \%$, specificity $97.11 \%$, diagnostic accuracy by $95 \%$, positive predictive value of $84.38 \%$, negative predictive value of $97.11 \%$, $(p=0.001)$ for prediction of maternal morbidity.

Conclusion: Modified early obstetrics warning system chart fulfills most of criteria of ideal screening tool making ita significant bedside screening tool which can predict obstetric morbidity and mortality in pregnant patients.
\end{abstract}

Keywords: Accuracy, Admission, Diagnostic, Intensive care, Modified early obstetric warning system, Maternal morbidity.

This is an Open Access article distributed under the terms of the Creative Commons Attribution License (https://creativecommons.org/licenses/by-nc/4.0/), which permits unrestricted use, distribution, and reproduction in any medium, provided the original work is properly cited.

\section{INTRODUCTION}

Childbearing is a normal life event for the majority of women. Healthy pregnancy, childbirth and postnatal recovery are usually ensured by physical adaptations which take place during pregnancy. However, unnoticeable signs of severe maternal illness are mostly masked by the increased cardiovascular reserves and other physiological changes that occurs in pregnant women, due to which recognition of impending maternal collapse difficult. It was well documented in a study that pregnant patients with serious illness, especially sepsis, may appear deceptively well before suddenly collapsing, often with little or no warning 1 . Factors which compromises wellbeing included; increased maternal age, obesityand women with complex medical conditions ${ }^{2}$.

The evidence-basedfact that physiological abnormalities precede critical illness led to development of early warning scoring systems ${ }^{3}$. The use of an early warning score that is modified for use in pregnant and postpartum women will helps in the early recognition, treatment and referral of women who have, or are developing, a critical illness, and is recommended. The modified early obstetric warning system (MEOWS)

Correspondence: Dr Uzma Urooj, Assistant Professor, Obs/Gynae Department, Pak Emirates Military Hospital Rawalpindi Pakistan

Received: 14 Feb 2020; revised received: 07 Jun 2020; accepted: 17 Jun 2020 chart is used in the postnatally up to the $6^{\text {th }}$ week following childbirth. It is likely that physiological changes will have returned to a pre-pregnant state at this stage and most pregnancy-related illness will be resolved ${ }^{4}$, MEOWS will help in early recognition.

MEOWS has been variably implemented for use by doctors and midwives, as it benefits in the management of women with established risk of morbidity ${ }^{5}$.

In a study by Ryan et al has showed that modified early obstetric warning system showed sensitivity $96 \%$ and specificity by $54 \%$ for prediction of maternal morbidity 6 .

In a study by Singh et al, has showed that modified early obstetric warning system showed $86.4 \%$ sensitive, $85.2 \%$ specific with a positive and negative predictive value of $53.8 \%$ and $96.9 \%$ respectively for prediction of maternal morbidity ${ }^{7}$.

No such study has been done before in our local population. Results of different studies have shown variability in results as shown above, therefore these results cannot be generalized on all populations. This study was conducted to get local evidence by determining the diagnostic accuracy of modified early obstetric warning system for prediction of maternal morbidity keeping ICU admission as gold standard. The objective of study was to determine the diagnostic accuracy of modified early obstetric warning system for 
prediction of maternal morbidity keeping intensive care admission as gold standard.

\section{METHODOLOGY}

This cross-sectional validation study was carried out at the department of Obstetrics/Gynecology, Pak Emirates Military Hospital, Rawalpindi, from October 2018 to April 2019. Prediction of maternal morbidity on MEOWS was defined as MEOWS score $>7$ during postnatal period up to the 6 th week postnatal.

Prediction of maternal morbidity on ICU admission was defined as women admitted to ICU in $<6$ weeks postnatal period for $>24$ hours due to postpartum hemorrhage, severe preeclampsia and eclampsia (any one). Table-I showing the MEOWS scoring system used.
Women were followed up to 6 weeks for maternal morbidity in term of ICU admission as per operational definition. First follow up was after 10 days postnatally and second follow up was carried out at 6 weeks postnatal. All the data regarding findings of Modified early obstetric warning system and ICU admission was noted by researcher herself on specially designed proforma. Identify specific triggers for responding to changes in the mother's vital signs and clinical condition and use protocols for responding to changes: Every time a set of observations is performed in either ante or postnatal women, MEOWS should be calculated and recorded in the observation chart. All women presenting to Triage who are having baseline observations carried out should have a MEOWS calculated and documented in the records every $12^{\text {th }}$ hourly. In order

Table-I: Modified early obstetric warning system scoring.

\begin{tabular}{l|c|c|c|c|c|c|c}
\hline Score & $\mathbf{3}$ & $\mathbf{2}$ & $\mathbf{1}$ & $\mathbf{0}$ & $\mathbf{1}$ & $\mathbf{2}$ & $\mathbf{3}$ \\
\hline Temperature & & $<35^{\circ} \mathrm{C}$ & & $35-37.4^{\circ} \mathrm{C}$ & & $37.5-39^{\circ} \mathrm{C}$ & $>39^{\circ} \mathrm{C}$ \\
\hline Systolic BP & Sc70 & $71-79$ & $81-89$ & $90-139$ & $140-149$ & $150-159$ & $2^{\prime}: 160$ \\
\hline Diastolic BP & & & Sc45 & $46-89$ & $90-99$ & $100-109$ & $2^{\prime}: 110$ \\
\hline Pulse & & Sc40 & $40-50$ & $51-100$ & $101-110$ & $111-129$ & $2^{\prime}: 130$ \\
\hline Respiratory rate & & Sc8 & & $9-14$ & $15-20$ & $21-29$ & $2^{\prime}: 30$ \\
\hline Alert, verbal, pain, unresponsive & & & & alert & Responds to voice & Responds to pain & Unconscious \\
\hline U/O ml per hour & $<10$ & $<30$ & & Not measured & & & \\
\hline
\end{tabular}

Triggering on MEOWS chart was defined as a single markedly abnormal observation (red trigger), or the combination of two simultaneous mildly abnormal observations (two yellow triggers). By using sensitivity and specificity calculator for sample size.

Sensitivity $=86.4 \%$, specificity $=95.2 \%$, prevalence $=16.61 \%$, confidence interval $=95 \%$. Absolute precision $=10 \%$, sample size $=205$ was calculated by using open EPI calculator. Non-probability convenience sampling was done. Inclusion criteria was women of age from 18-40 years, singleton pregnancy, postnatal period $<6$ weeks, history of hypertension and diabetes by medical record. Exclusion criteria included BMI $>28 \mathrm{~kg} / \mathrm{m}^{2}$ and refused for informed consent.

A 205 women from department of Obstetrics and Gynecology, Pak Emirates Military Hospital, Rawalpindi were included in the study following permission from ethics committee ER/49/A. Informed consent was taken from all patients/guardians explaining the benefits of study.

Modified early obstetric warning system was used antenatally in women fulfilling the inclusion criteria as per operational definition under the supervision of a consultant radiologist of three years post fellowship experience. to empirically derive an early-warning score to predict maternal death, physiologic data from obstetric patients were used.

Antenatal: Frequency of observations will depend on the nature of the admission or as indicated by the lead clinician. Full set of observations should be carried out twice daily at least 12 hours apart minimum. Delivery room: All women should have a set of MEOWS observations documented in the records on admission to Delivery room. Currently women who are in labour need not have MEOWS repeated. Regular observations should still be documented on the partogram as usual. However, a score attributable to the baseline observations on admission and recorded in the records. High risk Women receiving care on Delivery room should have the MEOWS score documented on the Mega chart. Normal MEOWS observation chart should be started once the Mega chart is no longer being used. The MEOWS chart should be initiated in recovery room by recovery practitioner prior to transfer to postnatal ward.

Postnatal ward: All women should have a full set of observations on admission to the postnatal ward and should have this repeated a minimum of 12 hours apart. A MEOWS score should be attributed to every 
set of observations. The frequency of observations will depend on the nature of the admission or as indicated by the lead practitioner. All obstetric inpatients must have a full set of observations and a MEOWS calculated at every level of transfer to a new area i.e., for example transfer from Recovery room to postnatal ward. The MEOWS chart used in one area should be transferred with the patient to the next area in order to help identify changes in trends of observations.

Data was entered and analyzed through SPSS-22. Mean \pm standard deviation was calculated for quantitative variables which included age, gestational age and parity. Frequency and percentages were calculated for qualitative variables like age groups. Sensitivity, specificity, positive predictive value, negative predictive value and diagnostic accuracy for Modified early obstetric warning system against ICU admission were calculated by using $2 \times 2$ model. Factors like age, gestational age and parity were controlled by stratification. Diagnostic accuracy was calculated, $p$-value of $\leq 0.05$ was considered statistically significant.

\section{RESULTS}

A total 205 women were included in the study. Age ranged from 18-40 years with mean of 31.585 \pm 3.31 years, mean gestational age was $37.595 \pm 1.84$ weeks and mean parity was $1.839 \pm 1$.59. Frequency and percentage of patients according to age groups was $18-30$ years $48.3 \%$ and $31-40$ years $51.7 \%$. MEOWS predicted $16.1 \%$ maternal morbidity and ICU admission predicted $15.6 \%$ maternal morbidity. MEOWS has shown sensitivity of $84.38 \%$, specificity $97.11 \%$, diagnostic accuracy by $95 \%$, PPV $84.38 \%$, NPV $97.11 \%$, ( $p=$ 0.001 ) for prediction of maternal morbidity (table-II).

Table-II: Comparison of MEOWS versus Intensive care unit admission for prediction of maternal morbidity.

Comparison of MEOWS versus Intensive care unit

admission for prediction of maternal morbidity.

\begin{tabular}{l|c|c|c}
\hline & Yes & No & \multirow{2}{*}{0.001} \\
\cline { 1 - 2 } Yes & $27(\mathrm{TP})$ & $5(\mathrm{FP})$ & \\
\hline No & $5(\mathrm{FN})$ & $168(\mathrm{TN})$ & \\
\multicolumn{3}{l|}{$\begin{array}{l}\text { TP: True positive, FP: False positive, FN: False negative, TN: True } \\
\text { negative }\end{array}$}
\end{tabular}

\section{DISCUSSION}

Morgan, Williams and Wright were amongst the pioneers who developed Early Warning System (EWS) in 1997 in UK8. These systems were developed in response to the fact that abnormalities in physiological parameters precede in patients who suffer a cardiopulmonary arrest ${ }^{9}$. Even good professionals fail to recognise early signs of maternal collapse according to Mat- ernal mortality case reviews and confidential enquiry into maternal and child health (CEMACH) 2003-05 report. Which led to a need for use of early warning system (EWS) for obstetric population also to predict maternal morbidity at an early stage ${ }^{10}$. Obstetric early warning system (ObsEWS) was established by intercollegiate maternal critical care group in order to detect deterioration earlier which can ultimately lead to improved maternal outcomes ${ }^{11}$. With some modifications in already existing early warning system (EWS), Maternal Early Obstetric Warning System (MEOWS) chart as recommended by CEMACH was specifically designed for pregnant patients. We carried out the study on 205 obstetric admissions which mainly included young (18-40 years) females. Another previous study tool done by Singh et al, also validated MEOWS toolin United Kingdom by studying 676 obstetric in patients who were from 20 weeks of gestation till 6 weeks postpartum ${ }^{12}$. Their socio-demographic characteristics were not available for comparison but different geographical areas, high economy, better literacy and better nutrition brought few differences while comparing our results with the study. In present study, $15.6 \%$ of the study population triggered which is almost similar $(30 \%)$ to the population in study by Singh et al. Being a developing country, low level of awareness, social taboos and tradition, difficult accessibility to health services in rural areas might be some of the causes for these associations in our study population. In our studyfew factors like elderly age, multiparity and low socio-economic class lead to increased obstetric morbidity which was not comparablewith another study done in 2014 by Soubra et al ${ }^{13}$. Baskett et al, reported delay in seeking care as well as delay in transfer as main factors leading to morbidity ${ }^{14}$. Poor transport facility and rural health infrastructure, custom and traditions contributes towards increase morbidity and mortality, as indicated in study carried out in our neighboring country ${ }^{15}$. There was a significantly higher proportion of interventions in triggered population in our study. It was also reported by Singh et al. (caesarean, ventouse or forceps delivery: $p<0.0001)^{12}$. The composite neonatal outcome was found to be poorer in triggered group but no comparison could be made due to lack of such data in available literature on MEOWS.

One of the advantages of this tool is value, cost effectiveness, safety to use, easily acceptable by community, accurate and validated as a screening tool. Validity is determined by Sensitivity and specificity whereas, accuracy is indicated by positive and negative predictive values. In our study, mostly all of the triggered 
patients were correctly identified as having morbidity and number of misleading triggers was less. There is no 'gold standard' obstetric early warning scoring system, although a number of hospitals in United Kingdom already using them. A survey in maternity units of UK in 2007 found that 30 (19\%) maternity units were regularly using an EWS in obstetric population yet only $9(6 \%)$ were using a system modified for parturient ${ }^{16}$. A sensitivity $84.38 \%$ and $97.11 \%$ of specificity in our study was comparable to the study by Ryan et al, apart from few contextual differences. In one of the retrospective studies done on 364 women with clinically diagnosed chorioamnionitis for prediction of sepsis, 6 different MEOWS had variable performance with 40$100 \%$ sensitivity, $4-97 \%$ specificity with a low positive predictive value of $<2-15 \%$ for all. Apart from this, the study also found simpler designs MEOWS to be more sensitive and useful ${ }^{17}$, which was similar to our study findings. Use of this bedside screening tool improved the quality of care, decrease morbidity and mortality and eventually lead to increased health care practices for our patients ${ }^{18}$. The "track and trigger" of physiological parameters can reduce morbidity and mortality by early recognition so as to prevent cascade of drastic events ultimately leading to better maternal care ${ }^{19}$, as shown in study done by Paternina-Caicedo et al.

Recommendation is to use it routinely in every obstetric unit in order to timely recognize any critical illness and periodic documentation of physiological parameters. Further studies should be carried out to further validating the tool.

\section{CONCLUSION}

For predicting obstetric morbidity, MEOWS chart can be a practical bedside screening tool as it meets most of the criteria of an ideal screening tool in pregnant patients in our context. It should be routinely used as bedside screening tool for early recognition of any life threatening situation in order to decrease maternal morbidity and mortality.

\section{CONFLICT OF INTEREST}

This study has no conflict of interest to be declared by any author.

\section{REFERENCES}

1. Vivekanantham S, Mudalige N, Suri V, Kamaledeen A, Law P. Maternal and fetal death following group a streptococcal meningitis in mid-term pregnancy. Case Rep Obstet Gynecol 2014; 13(4): 117-20.
2. Lee S. Risk perception in women with high-risk pregnancies. Br J Midwifery 2014; 22(1): 8-13.

3. Wuytack F, Meskell P, Conway A, Mc Daid F, Santesso N, Hickey FG, et al. The effectiveness of physiologically based early warning or track and trigger systems after triage in adult patients presenting to emergency departments: a systematic review. BMC Emerg Med 2017; 17(1): 38-42.

4. Soma-Pillay P, Catherine NP, Tolppanen H, Mebazaa A, Tolppanen $\mathrm{H}$, Mebazaa A. Physiological changes in pregnancy. Cardiovasc J Afr 2016; 27(2): 89-92.

5. Mackintosh N, Watson K, Rance S, Sandall J. Value of a modified early obstetric warning system (MEOWS) in managing maternal complications in the peripartum period: an ethnographic study. BMJ Qual Saf 2014; 23(1): 26-34.

6. Ryan HM, Jones MA, Payne BA, Sharma S, Hutfield AM, Lee T, et al. Validating the performance of the modified early obstetric warning system multivariable model to predict maternal intensive care unit admission. J Obstet Gynaecol Canada 2017; 39(9): 728-33.

7. Singh A, Guleria K, Vaid NB, Jain S. Evaluation of maternal early obstetric warning system (MEOWS chart) as a predictor of obstetric morbidity: a prospective observational study. Eur J Obstet Gynecol Reprod Biol 2016; 207(1): 11-7.

8. Morgan R, Lloyd-Williams F, Wright MM, Morgan-Warren RJ. An early warning scoring system for detecting developing critical illness. Med 1997; 18(6): 31-34.

9. Schein RMH, Hazday N, Pena M, Ruben BH, Sprung CL. Clinical antecedents to in-hospital cardiopulmonary arrest. Chest. 1990; 98(6): 1388-92.

10. Maguire PJ, Power KA, Turner MJ. The maternal early warning criteria: a proposal from the National Partnership for Maternal Safety. Obstet Gynecol 2015; 125(2): 493-94.

11. Quinn AC, Meek T, Waldmann C. Obstetric early warning systems to prevent bad outcome. Curr Opin Anesthesiol 2016; 29(3): 268-72.

12. Singh S, Mc Glennan A, England A, Simons R. A validation study of the CEMACH recommended modified early obstetric warning system (MEOWS). Anaesth 2012; 67(1): 12-8.

13. Soubra SH, Guntupalli KK. Critical illness in pregnancy: an overview. Crit Care Med 2005; 33(10): 48-55.

14. Baskett TF, O'Connell CM. Maternal critical care in obstetrics. J Obstet Gynaecol Canada 2009; 31(3): 218-21.

15. Bajwa SK, Bajwa SJS, Kaur J, Singh K, Kaur J. Is intensive care the only answer for high risk pregnancies in developing nations. J Emergencies, Trauma Shock 2010; 3(4): 331-34.

16. Friedman AM, Campbell ML, Kline CR, Wiesner S, D'Alton ME, Shields LE. Implementing obstetric early warning systems. Am J Perinatol Reports 2018; 8(2): 79-84.

17. Edwards SE, Grobman WA, Lappen JR, Winter C, Fox R, Lenguerrand $\mathrm{E}$, et al. Modified obstetric early warning scoring systems (MOEWS): validating the diagnostic performance for severe sepsis in women with chorioamnionitis. Am J Obstet Gynecol 2015; 212(4): 536-1.

18. Umar A, Ameh CA, Muriithi F, Mathai M. Early warning systems in obstetrics: A systematic literature review. PLoS One 2019; 14(5): :e0217864.

19. Paternina-Caicedo A, Miranda J, Bourjeily G, Levinson A, Dueñas C, Bello-Muñoz C, et al. Performance of the obstetric early warning score in critically ill patients for the prediction of maternal death. Am J Obstet Gynecol 2017; 216(1): 58-1. 